\title{
Plasmon Energy Mapping in Aluminum and Indium with Sub-Nanometer Resolution
}

\author{
Brian Zutter ${ }^{1}$, Matthew Mecklenburg ${ }^{2}$, and B. C. Regan ${ }^{1}$ \\ 1. Department of Physics and Astronomy \& California NanoSystems Institute, University of California, \\ Los Angeles, CA, USA \\ 2. Center for Electron Microscopy and Microanalysis, University of Southern California, Los Angeles, \\ CA, USA
}

In the free electron model, the plasmon energy is proportional to the square root of the valence electron density. Using scanning transmission electron microscopy (STEM) and electron-energy loss spectroscopy (EELS) to measure plasmon energies, one can generate electron density maps with sufficient resolution to reveal atomic-scale features [1]. We use STEM EELS to map the plasmon energy in aluminum and indium films on a $5 \mathrm{~nm}$-thick silicon nitride membranes. Using an aberration-corrected STEM, we have acquired spectrum images with $80 \mathrm{pm}$ spatial resolution. In both metals we see atomic periodicity in the plasmon energy signals.

Indium and aluminum films were e-beam evaporated onto electron-transparent, silicon nitride windows. High-resolution annular bright field (ABF) STEM images reveal the respective atomic lattices (see Figure $1 \mathrm{~A}$ and $\mathrm{C}$ ). EELS spectra were acquired using a cold field emission gun (FEG) and GIF Quantum-965. An accelerating voltage of $80 \mathrm{kV}$ was used for aluminum, while $300 \mathrm{kV}$ was used for indium. The zero loss peak (ZLP) and the plasmon peak of the spectra were fit to Gaussian functions, and the difference between the center values was taken to be the plasmon energy (Figure $1 \mathrm{~B}$ and D). The mean plasmon energies were measured to be $11.47 \pm .01 \mathrm{eV}$ and $15.084 \pm .009 \mathrm{eV}$ in indium and aluminum respectively. (See Figure $2 \mathrm{~A}$ for representative, single pixel spectra.)

The fast Fourier transforms (FFT) of the ABF and plasmon energy images are shown in the bottom row of Figure 1. The FFTs of ABF images $1 \mathrm{~A}$ and $1 \mathrm{C}$ clearly show six first-order reciprocal lattice points. All six first-order reciprocal-lattice points are visible in the FFT of the indium plasmon energy map (1B), while only two first-order points are visible in the FFT of the aluminum plasmon energy map (1D). The lattice is also clearly visible in a line profile measurement of the indium plasmon energy real space image, and evident, though less obviously so, in the corresponding aluminum line profile (Figure 2B).

The evident lattice periodicity in the plasmon energy map is a surprising result given the delocalization of the plasmon. For instance, the plasmon delocalization distance is approximately $3 \mathrm{~nm}$ for the $15.2 \mathrm{eV}$ $\mathrm{Al}$ plasmon at $80 \mathrm{kV}$ [2], more than a factor of ten larger than the nearest-neighbor spacing. It is not yet clear whether the observed periodicity represents a real measurement of the atomic-scale variations in the electron density, or a residual, systematic signal from resulting from imperfect curve fitting. In aluminum the Thomas-Fermi (screening) length is approximately $7 \mathrm{~nm}$, meaning that the electron density does vary on an atomic scale [3]. The better visibility of the lattice periodicity in the plasmon energy in indium relative to aluminum can be understood by noting that the electron wavelength changes from $4.2 \mathrm{pm}$ at $80 \mathrm{kV}$ to $2 \mathrm{pm}$ at $300 \mathrm{kV}$, thereby decreasing the delocalization distance [3]. If this effect proves real, it may be possible to use STEM and EELS to map the plasmon energy around a point or interstitial defect in a crystal lattice and thereby measure changes in the local electron density. 
References:

[1] M Mecklenburg et al, Science 347 (2015), p. 629-632.

[2] R Egerton, Reports on Progress in Physics. 72 (2009), p. 25.

[3] P Schattschneider, "Fundamentals of Inelastic Electron Scattering” (Springer-Verlag) p. 118.

[4] This work was supported by FAME, one of six centers of STARnet, a Semiconductor Research Corporation program sponsored by MARCO and DARPA, by National Science Foundation (NSF) award DMR-1611036, and by NSF STC award DMR-1548924. Data presented were acquired using a Grand ARM at the JEOL factory in Tokyo, Japan.
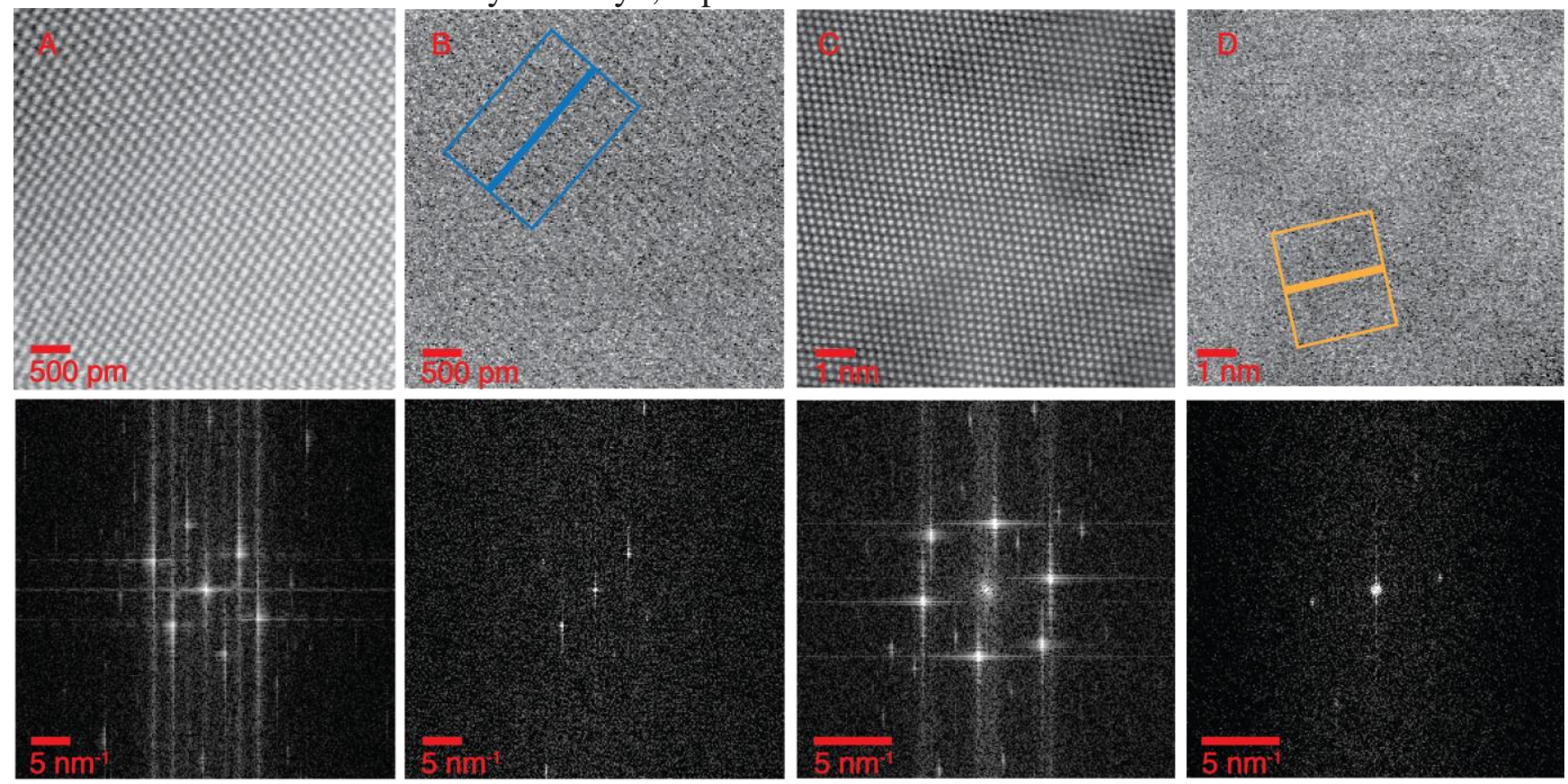

Figure 1. Top row are real-space images, and the bottom row gives respective fast Fourier transforms. A) $\mathrm{ABF}$ of a $100 \mathrm{~nm}$ indium film. B) Plasmon energy map of the same field of view as A). C) ABF of a $60 \mathrm{~nm}$ aluminum film. D) Plasmon energy map of the same field of view as C)
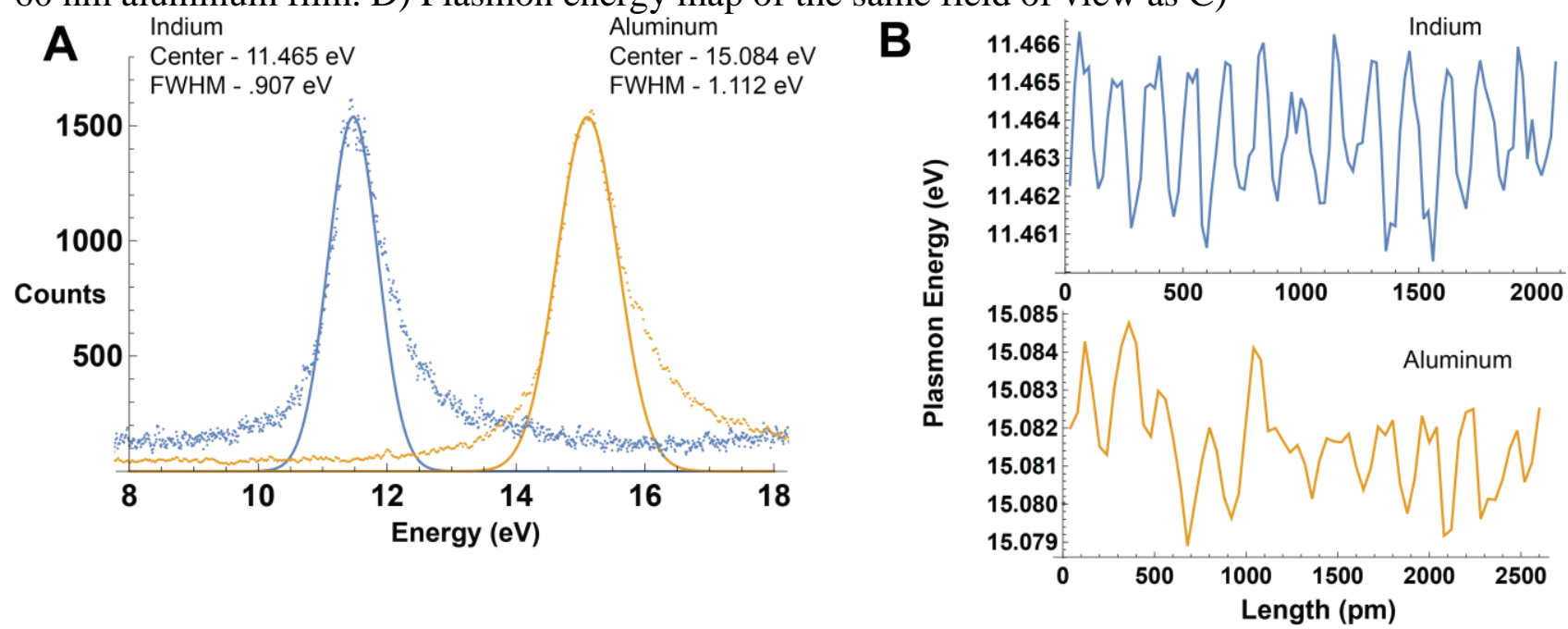

Figure 2. A) Representative EELS spectra of indium and aluminum. B) Line profiles of the indium plasmon energy map (yellow) and the aluminum plasmon energy map (blue). The line profiles were taken from the blue and yellow regions in Figure 1 and averaged across the box perpendicular to the line direction. 